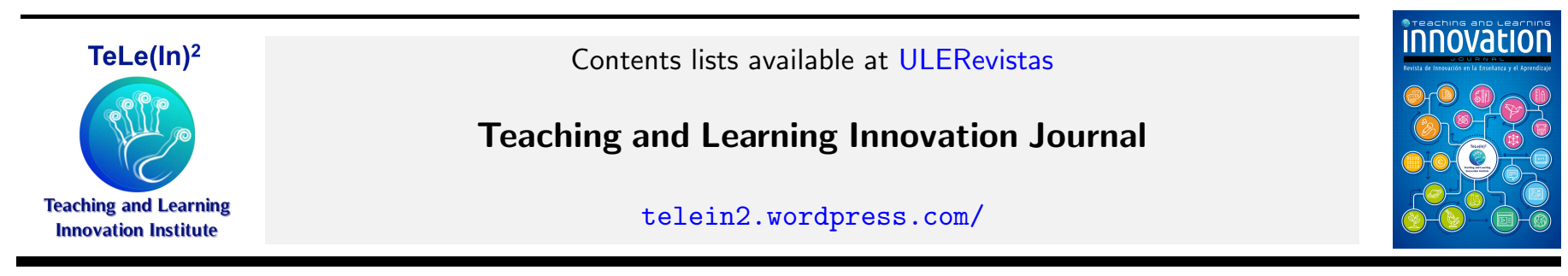

\title{
Formación a distancia en materia de ingeniería de soldadura: enfoque multidisciplinar mediante modelos 3D. \\ E-learning in welding engineering: a multidisciplinary approach using 3D models.
}

\author{
Manuel Rodríguez-Martínª,c , Pablo Rodríguez-Gonzalvez ${ }^{\mathrm{b}}$ \\ ${ }^{a}$ Grupo de Investigación TIDOP. Escuela Politécnica Superior de Ávila. Universidad de Salamanca. Ávila, Spain \\ ${ }^{b}$ Departamento de Tecnología Minera, Topografía y Estructuras. Universidad de León. León, Spain \\ ${ }^{c}$ Facultad de Ciencias y Artes. Universidad Católica de Ávila. Ávila, Spain
}

\begin{abstract}
El presente trabajo describe una nueva metodología docente basada en las últimas investigaciones científicas orientadas a la reconstrucción tridimensional de soldaduras, las cuales permiten la generación de paquetes de trabajo dirigidos a la adquisición de competencias propias de actividades de laboratorio de soldadura sin necesidad de que exista un desplazamiento físico al mismo. Esta metodología, que puede ser catalogada dentro de las basadas en laboratorios virtuales (LVs), es de aplicación en programas semipresenciales, e-learning o también puede utilizarse como material de apoyo a los programas presenciales, principalmente en las titulaciones relacionadas con la ingeniería industrial, naval y aeronáutica, así como las ingenierías más relacionadas con la geomática y la informática. La distribución de los paquetes es de fácil implementación a través de plataformas virtuales de formación y el trabajo con los modelos puede realizarse con software libre o gratuito, sin necesidad de costes adicionales para las entidades de formación que los utilicen.

Abstract- The present work describes a new teaching methodology based on the latest research oriented to the threedimensional reconstruction of welds that allows the generation of work packages aimed at the acquisition of competences specific to welding lab activities without the need for a physical same. This methodology, which can be cataloged within the methodologies based on virtual laboratories (LVs), is applicable in e-learning programs or can also be used as support material for face-to-face programs, mainly in the degree programs related to Industrial, naval and aeronautical engineering, as well as engineering related to geomatics and computer science. The distribution of the packages is easy to implement through virtual training platforms and the work with the models can be done with open or free software, without the need of additional costs for the training entities that use them.
\end{abstract}

Keywords: e-learning, laboratorios virtuales (LVs), ensayos no destructivos (END), inspección de soldadura, educación superior e-learning, virtual laboratories (VLs), Non-destructive testing (NDT), welding inspection, high education

\section{Introducción}

El uso de técnicas orientadas a la adquisición de competencias en materia de laboratorios mediante virtualización (LVs) está adquiriendo una mayor relevancia en enseñanzas superiores de ámbito universitario (Vergara et al., 2016). Son varios los trabajos que lo abordan para titulaciones de ciencia e ingeniería (Heradio et al., 2016; Vergara et al, 2014). Algunos de ellos abordados con criterios de bajo

Email addresses: ingmanuel@usal.es (Manuel Rodríguez-Martín), p.rodriguez@unileon.es (Pablo Rodríguez-Gonzalvez) coste (Rositer 2016), siendo también destacables aquellos trabajos orientados a las enseñanzas medias (Cerezo y Sastrón, 2015).

Una posible razón por la cual este tipo de metodologías de enseñanza se está extendiendo tanto horizontal como verticalmente radica en el alto coste de ciertas instalaciones de laboratorio, circunstancia que, en numerosas ocasiones, actúa como una intransitable frontera que impide el acceso a la formación práctica y a los contenidos propios de disciplinas que atesoran una alta carga de laboratorio, de taller o la necesidad de instalaciones específicas de formación. Ello puede ocasionar un impedimento para 
los estudiantes de programas especializados de naturaleza científica y técnica, tanto en el ámbito universitario como en el empresarial. Otra razón que justifica el diseño metodoló basado en laboratorios virtuales descansa en la necesidad de aportar una formación a distancia que no obligue al desplazamiento presencial de los alumnos a las instalaciones de laboratorio, sobre todo en aquellos programas de naturaleza semipresencial o a distancia (Muñoz-Nieto, 2014; Rodríguez-Gonzálvez et al., 2017). Cada vez son más las universidades y entidades empresariales de enseñanza que ofertan estudios en este tipo de modalidades, debido al aumento desde hace más de 10 años de la demanda para este tipo de programas y al aumento del volumen de alumnos que ponen interés en este tipo de enseñanza (Calvo Verdú, 2006).

Además, con el uso de tecnologías orientadas a la sustitución de laboratorios físicos se consiguen otros objetivos docentes como se indica en Vergara et al. (2014). Por un lado, se puede lograr solventar la problemática que supone tener grupos extensos en contextos de laboratorios de alta especialización, que tiene en ocasiones como consecuencia una mayor aglomeración de alumnado entorno a elementos tangibles (máquinas, herramientas, equipos, muestras, etc.). Por otro lado se corrobora que en contextos formativos de laboratorio, ciertos detalles de las metodologías del trabajo o del funcionamiento de los equipos no son apreciados de manera efectiva por los alumnos debido a la rapidez de ejecución de una determinada actividad práctica o debido al reducido tamaño de la zona donde se realiza la actividad práctica. A este respecto, los contextos virtuales para la enseñanza de laboratorio, taller o instalaciones permiten diseñar ad-hoc la zona de interés, interactuar dentro de ella, dejar al alumno todo el tiempo que necesite para adquirir los conocimientos y competencias y evitar cualquier complicación derivada de la manipulación de sustancias, máquinas o equipos de alta peligrosidad o de potencial contaminante.

Los ensayos habitualmente utilizados en soldadura son la inspección visual, las partículas magnéticas, los líquidos penetrantes, las corrientes eléctricas, los ultrasonidos y los métodos radiográficos. Un primer enfoque para la posible virtualización del ensayo no destructivo de soldadura por la técnica ultrasónica ha sido realizado en (Vergara et al., 2016). Sin embargo a día de hoy no hay constancia de la existencia de métodos remotos de formación para el ensayo de inspección visual, siendo este uno de los ensayos más utilizados en inspección de soldadura y uno de los que más pericia y experiencia precisa por parte del inspector, al no basarse totalmente en métodos cuantitativos ni en el auxilio de equipos tecnológicos sino la mayor carga del procedimiento recae en la interpretación cualitativa por parte del inspector de la calidad superficial de la soldadura, así como en mediciones que, en ocasiones, no son realizadas con la debida precisión debido a la ausencia de métodos objetivos de medida.

Según se indica en Rodríguez-Martín et al. (2015), la inspección visual de las soldaduras es una prueba del todo importante, aplicada a la detección de imperfecciones y defectos superficiales, los cuales junto con los defectos intergioms constituyen la defectología de más frecuente aparición en las soldaduras (AWS, 2004). La inspección visual sirve para dar una visión general de conjunto sobre el estado global de la soldadura y hacer una detección temprana de posibles defectos e imperfecciones y, como toda la mayoría de ensayos de calidad en soldaduras, está regulada por una normativas de calidad. Se trataría, por tanto, de una especie de ensayo "de cabecera" que, en ocasiones, por sí mismo puede garantizar la calidad de una soldadura o que puede tener como resultado el rechazo parcial o total de la misma o plantear la necesidad de aplicación de técnicas más precisas que permitan abordar la defectología interior.

Para formar a un profesional en el ensayo de inspección visual de soldadura, la instrucción se debe adecuar a los estándares internacionales donde quedan tasadas una serie de patologías, así como los criterios de aceptación o rechazo de la soldaduras (como por ejemplo: ISO-5817:2009; ISO 6520-1:2007); criterios que, por otro lado, se basan en mediciones geométricas realizadas sobre la propia soldadura. Las entidades que imparten formación de este tipo deben contar con numerosos especímenes (Fig. 1) que permitan al alumno conocer las diferentes tipologías de soldeo, así como los principales tipos de defectos e imperfecciones. Estos especímenes se comercializan para formación y su coste es alto, dado que cada uno de ellos tiene que tener un tipo de defecto concreto para que el alumno pueda contextualizarlo dentro del abanico de defectología dispuesta en la norma. El coste aproximado de cada espécimen está entre los 150 y los 2000 euros, en función del tipo de soldadura (soldadura a tope, soldadura en T, L, doble T, soldadura de tuberías, soldadura de estructuras basadas en tubos, etc.) y también del material (principalmente acero o aluminio). El alto coste de cada espécimen, unido a la necesidad de tener varios distintos (dado que, como se ha dicho, cada uno expone un defecto concreto y existen numerosos tipos de defectos) hace que contar con este tipo de materiales en los laboratorios o talleres orientados a la formación de soldadura sea excesivamente caro y fuera del alcance de ciertas instituciones que abordan formación de este tipo.

Los programas en los que normalmente se abordan los contenidos de inspección de soldadura dentro de los programas de sus asignaturas son los relacionados con la ingeniería mecánica, las tecnologías industriales y la ingeniería aeroespacial y naval. Sin embargo, un inspector de soldadura es normalmente un profesional con formación en esas ingenierías pero que, además, cuenta con una dilatada experiencia en procesos de soldadura e ingeniería de materiales que ha pasado diferentes exámenes con el fin de obtener un certificado de inspector para cada metodología concreta (AWS, 2006).

La reconstrucción tridimensional de objetos cercanos es una técnica ya asentada y democratizada con ciertos dispositivos de baja precisión. Los métodos que aportan una alta fidelidad geométrica (ACMM en inglés, de sus siglas 


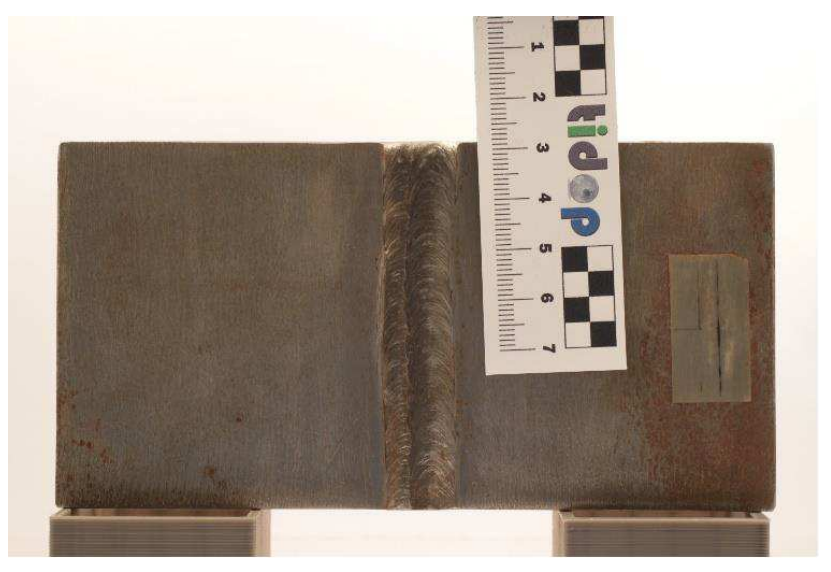

Figure 1: Fotografía de espécimen de soldadura TIG de acero al carbono con defecto de porosidad interna disponible en laboratorio real. Para que el lector se haga una idea, este pequeño espécimen puede tener un precio de mercado cercano a los 200 euros y solo representa un tipo de material, un tipo de metodología de soldeo y un tipo de defecto.

Articulated Coordinate Measure Machine) siguen siendo costosos y, en ocasiones, no están disponibles en los laboratorios de las escuelas que imparten titulaciones cuyo estudio debería ser objeto de las mismas: principalmente los grados en ingeniería geomática y topografía, etc.

Aplicando la reconstrucción tridimensional a los especímenes de soldadura se pueden obtener modelos tridimensionales que recreen con fidelidad la compleja geometría de una soldadura y que sirvan, tanto para la enseñanza dentro de programas o titulaciones orientadas a formar inspectores de inspectores de soldadura (ámbito industrial), como para aquellos otros donde se aborden las técnicas geomáticas de escaneo tridimensional. En esta línea se desarrollará la presente comunicación.

\section{Contexto}

En el ámbito de los laboratorios virtuales para la formación remota, existen varios trabajos que abordan herramientas didácticas virtuales en el ámbito de las ciencias de la salud (Osborne et al., 2015; Mastmeyer et al., 2016) pero en el ámbito de la formación en inspección visual de soldadura, no se tiene constancia de ninguna herramienta educativa con aplicación al ámbito de la ingeniería que permita trabajar sobre modelos virtuales. Sin embargo, a nivel tecnológico, la reconstrucción tridimensional de soldaduras para labores de inspección remota y/o automática es un campo de investigación en desarrollo a día de hoy, en el que participan activamente los autores de la presente comunicación.

\section{Descripción}

La secuencia seguida para el diseño metodológico (Fig. 2) comienza con un estudio de virtualización que analice el alcance de la metodología, las titulaciones o modalidades de estudio potenciales para su aplicación, etc.

Una vez hecho esto se aborda el proceso técnico de virtualización, basado en la generación de modelos utilizando tecnología de escaneo láser e imagen fotográfica de alta resolución. Después, el material resultante se somete a una evaluación para analizar el cumplimiento de los requerimientos y necesidades establecidos en la primera fase para, finalmente, proceder al diseño del paquete de distribución.

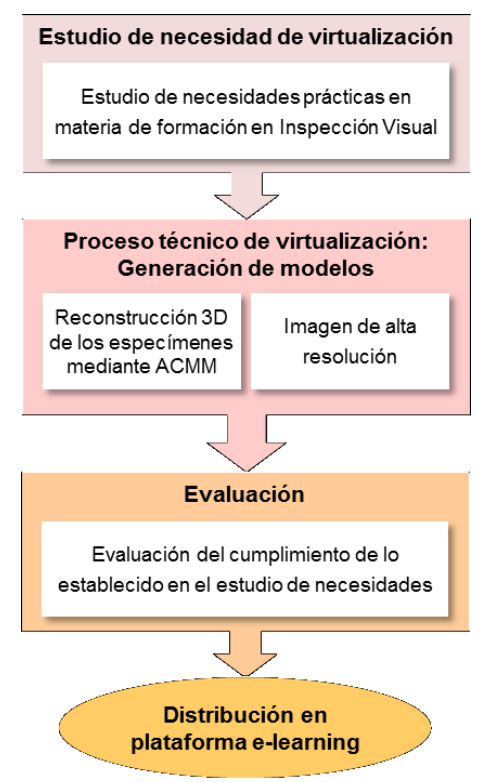

Figure 2: Diagrama de flujo del proceso de generación y distribución de los paquetes de trabajo.

\section{A. Estudio de necesidades de virtualización}

Las necesidades de virtualización se abordan contando con el objetivo principal: aportar un aprendizaje al alumno en materia de ensayo de soldaduras y de reconstrucción tridimensional de manera remota y sin necesidad de desplazamiento a laboratorios, ni de posesión de especímenes físicos. A este respecto, primeramente, se deberá evaluar la ventaja en términos de aprendizaje para los diferentes tipos de formación (Tabla 1).

Todos los grupos necesitan los modelos tridimensionales para adquirir los conocimientos, competencias y habilidades propuestas en la Tabla 1. Sin embargo, el G1 y el G3, además de los modelos tridimensionales (sin textura), necesitarían un aporte extra en forma de imágenes para que puedan analizar diferentes patologías e imperfecciones que pueden no detectarse en el modelo tridimensional, tales como oxidaciones, inclusiones de escoria superficiales, manchas de pintura, etc. De tal forma que los paquetes de materiales pueden ser asimétricos para una 
Table 1: Diferentes necesidades formativas para los diferentes grupos de potenciales destinatarios de los paquetes de trabajo.

\begin{tabular}{|c|c|c|}
\hline \multicolumn{3}{|c|}{ Necesidades formativas detectadas } \\
\hline $\begin{array}{l}\text { G1. Títulos ofi- } \\
\text { ciales de ingeniería } \\
\text { de la rama indus- } \\
\text { trial, naval y aeropa- } \\
\text { cial. }\end{array}$ & $\begin{array}{l}\text { G2. Títulos oficiales } \\
\text { de ingeniería de la } \\
\text { rama geomática e in- } \\
\text { formática. }\end{array}$ & $\begin{array}{l}\text { G3. Formación es- } \\
\text { pecífica no univer- } \\
\text { sitaria para certifi- } \\
\text { cación de inspectores } \\
\text { VT. }\end{array}$ \\
\hline $\begin{array}{l}\text { Conocer los difer- } \\
\text { entes tipos de sol- } \\
\text { dadura con arreglo al } \\
\text { proceso de soldeo. }\end{array}$ & $\begin{array}{l}\text { Conocer diferentes } \\
\text { algoritmos de gen- } \\
\text { eración de modelos } \\
\text { 3D (tipos de mallado } \\
\ldots \text {. ) }\end{array}$ & $\begin{array}{l}\text { Conocer los princi- } \\
\text { pales defectos e im- } \\
\text { perfecciones superfi- } \\
\text { ciales en soldadura. }\end{array}$ \\
\hline $\begin{array}{l}\text { Conocer las difer- } \\
\text { entes tipologías de } \\
\text { uniones soldadas, así } \\
\text { como las diferentes } \\
\text { disposiciones de sol- } \\
\text { deo. }\end{array}$ & $\begin{array}{l}\text { Conocer los efectos } \\
\text { de los diferentes ma- } \\
\text { teriales y acabados } \\
\text { superficiales en los } \\
\text { modelos 3D. }\end{array}$ & $\begin{array}{l}\text { Conocer las rela- } \\
\text { ciones geométricas } \\
\text { aportadas por la } \\
\text { normativa para } \\
\text { analizar la severidad } \\
\text { de los diferentes } \\
\text { tipos de defectos e } \\
\text { imperfecciones. }\end{array}$ \\
\hline $\begin{array}{l}\text { Conocer los princi- } \\
\text { pales defectos e im- } \\
\text { perfecciones superfi- } \\
\text { ciales en soldadura. }\end{array}$ & $\begin{array}{l}\text { Conocer la influencia } \\
\text { y repercusión de los } \\
\text { algoritmos de mal- } \\
\text { lado y simplificación } \\
\text { en la precisión fi- } \\
\text { nal de modelos sub- } \\
\text { milimétricos. }\end{array}$ & $\begin{array}{l}\text { Conocer las fases del } \\
\text { procedimiento de in- } \\
\text { spección. }\end{array}$ \\
\hline $\begin{array}{l}\text { Conocer las rela- } \\
\text { ciones geométricas } \\
\text { aportadas por la } \\
\text { normativa para } \\
\text { analizar la severidad } \\
\text { de los diferentes } \\
\text { tipos de defectos e } \\
\text { imperfecciones. }\end{array}$ & $\begin{array}{l}\text { Trabajar con casos } \\
\text { reales de estudio con } \\
\text { requisitos de pre- } \\
\text { cisión y resolución } \\
\text { submilimétrica. }\end{array}$ & $\begin{array}{l}\text { Redactar los in- } \\
\text { formes preceptivos } \\
\text { para plasmar doc- } \\
\text { umentar el ensayo } \\
\text { visual de soldadura. }\end{array}$ \\
\hline
\end{tabular}

mejor adaptación a las necesidades formativas de cada titulación o tipo de estudio (Fig. 3).

\section{B. Generación de modelos}

La generación de modelos se realizará utilizando una máquina metrológica articulada (ACMM), concretamente el modelo Hexagon Romer Absolute Arm 7325 SI 3D scanner. Este tipo de dispositivo de orientación metrológica, está equipado con un sensor de barrido láser que recompone tridimensionalmente superficies cercanas por el método de la triangulación láser con una precisión de \pm 49 micrómetros (Rodríguez Gonzalvez et al., 2016) y que aporta métrica real (sensor activo). Es difícil encontrar soluciones en el mercado que aporten una fidelidad geométrica mayor en la reconstrucción de superficies complejas y, que, a su vez, permitan un escaneo sencillo y rápido de objetos pequeños como son las soldaduras. Existen otros métodos de escaneo de modelos tridimensionales basados en sensores activos, pero ninguno aglutina las facilidades en cuanto a versatilidad, flexibilidad, operatividad y precisión como el ACMM.

El procedimiento de escaneo con ACMM es tan sencillo como desplazar el sensor láser a través de la soldadura (Fig. 4) para que el haz realice un barrido de la soldadura y se vaya generando su modelo tridimensional. El resultado (Fig. 5) es una nube de puntos mallada de alta precisión que muestra con gran fidelidad, la morfología superficial de la soldadura.
El modelo láser de alta precisión refleja la geometría de la soldadura mientras que la información radiométrica es dada por la imagen fotográfica tomada con una cámara Canon EOS 700D y con una lente macro de $50 \mathrm{~mm}$ de focal. La información geométrica del modelo posiblemente sea la que más interés tenga de cara al ámbito formativo en inspección de soldadura según los estándares internacionales, sin embargo, existen patologías en soldadura que se detectan desde la cromaticidad de la misma (e.g. corrosión, pequeñas salpicaduras que escapan de la resolución del escáner, etc.). La textura de la imagen no es obtenida con el ACMM (que es un sistema metrológico puro). Se podría plantear una texturización del modelo puro a partir de fotografías para generar modelos fotorealísticos, pero esta posibilidad sería compleja desde el punto de vista computacional, se debería de plantear para cada caso de estudio una estrategia concreta (poco estandarizable) y los resultados podrían no ser adecuados. Por ello se considera que la mejor opción a día de hoy, la más óptima, operativa $\mathrm{y}$ viable consiste en incluir junto a los modelos tridimensionales, imágenes fotográficas en alta resolución, a fin de que el alumno tenga un paquete de trabajo completo que le permita su formación en el ámbito de la inspección de soldadura.

Las imágenes de 18 megapíxeles de resolución fueron tomadas en condiciones de luminosidad de laboratorio, pero sin ningún tipo de iluminación extra para no enmascarar las posibles patologías e imperfecciones superficiales de los especímenes. A tal fin fue necesario emplear una apertura pequeña del obturador (f/8), tiempos de exposición lentos (superiores al segundo) y la menor sensibilidad ISO posible para evitar altos niveles de ruido. Se demostró experimentalmente que estas condiciones de captura aportaban una óptima fidelidad cromática del espécimen y la resolución era suficientes para ver con nitidez detalles submilimétricos como algunas pequeñas salpicaduras (Fig. 4). La disposición de toma de las fotografías permite una rectificación en dos dimensiones, a fin de poder tomar medidas sobre la imagen. Lógicamente, este tipo de medida sobre imagen otorga menos precisión que la medida sobre el modelo tridimensional pero puede servir al alumno para contrastar realidades métricas o para medir imperfecciones que solo se vean en las imágenes (por ejemplo, imperfecciones de detección cromática como las oxidaciones).

\section{Evaluación.}

Una vez obtenido el resultado del proceso de generación de modelos tridimensionales y de las imágenes de alta resolución, se torna como necesario estudiar si el resultado cumple con los requerimientos previamente establecidos en el apartado A para la titulación en cuestión. En este caso queda totalmente acreditada la calidad métrica de los modelos, así como su fidelidad cromática y, por ende, la correcta adecuación a los requisitos de calidad que se esperaba del material diseñado.

D. Diseño y distribución del material docente.

El material docente se debe preparar de tal manera que el alumno objeto del mismo reciba la información necesaria 
para poder adquirir las competencias establecidas según su grado de estudios. A este respecto y en consonancia con lo dispuesto en la Tabla 1 se proponen 3 paquetes de material (Fig. 3). Uno para cada uno de los potenciales alumnos. Los tres paquetes comparten los mismos modelos y las mismas imágenes en aras de conseguir una mayor optimización en el diseño y distribución del material. Lo que los diferencia es el material documental / bibliográfico que se adjunta dentro de los paquetes. En ellos versarán tanto los contenidos teóricos de estudio, como los contenidos de autoevaluación y las actividades a realizar por el alumno para adquirir las competencias en materia de inspección visual de soldadura o de reconstrucción tridimensional mediante escáner láser. Los paquetes de material deberán ser comprimidos en un formato ZIP, RAR o similar para difundirlos por la plataformas de e-learning de la que disponga la institución de enseñanza (tipo Moddleß), Blackboard®, etc.).

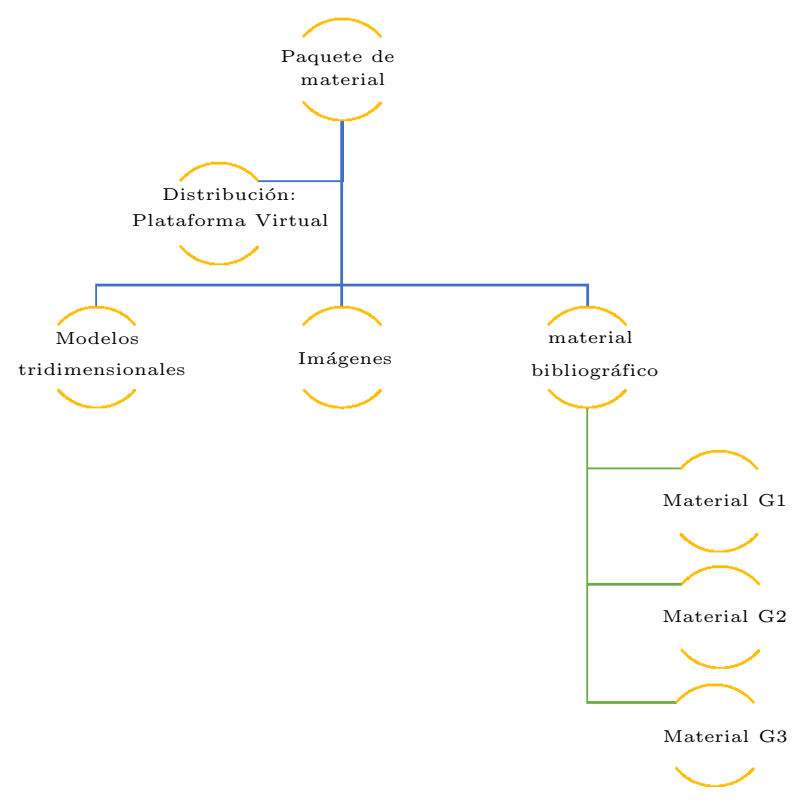

Figure 3: Composición de los paquetes de trabajo.

\section{Resultados}

Los resultados de la aplicación de la metodología propuesta se materializarán, como ya se ha indicado, en paquetes de trabajo elaborados para grupo de interés (Tabla 1). A este respecto se muestran (Fig. 4-7) los outputs del procedimiento. Por un lado, los modelos de soldadura en tres dimensiones de alta precisión obtenidos con ACCM, sobre los cuales pueden ser visualizados con el software libre CloudCompare [17]. Este software, además de visualizar el resultado $3 \mathrm{D}$, permite tomar mediciones sobre el modelo para que el alumno pueda elaborar las tareas que se la hayan propuesto, pudiendo medir, incluso con más precisión de posicionamiento que si estuviera en el laboratorio con un pie de rey o con instrumentos físicos orientados a la toma de medidas en soldadura. También es posible el seccionamiento del modelo para analizar con precisión sus medidas geométricas y analizar si estas son acordes con los criterios de calidad dispuestos en la normativa internacional (e.g: ISO-5817: 2009, ISO 6520-1:2007) o, incluso utilizar un procedimiento de computación de distancias para sacar mapas de profundidad que permitan analizar las zonas con sobre-espesor (Fig. 6) (Rodríguez-Martín et al., 2017). Todas estas labores son de interés, tanto para el G1 como para el G3, al ser competencias básicas que debe tener todo inspector de soldadura el saber analizar visualmente la calidad del cordón de soldadura y tomar las medidas necesarias sobre él para estudiar si se cumplen los requerimientos de calidad establecidos en los estándares internacionales que, por otra parte, podrían estar incluidos en el material bibliográfico del paquete de trabajo para los grupos G1 y G3. Respecto al grupo G2, dado que dentro de sus competencias se enmarca la generación de los modelos 3D, la presencia de resúmenes sintetizados de los requisitos de los estándares internacionales permitirá que los estudiantes puedan valorar el grado de consecuencia de resultados, errores propagados en su generación, y priorizar los aspectos más relevantes que requerirán los usuarios finales en su actividad profesional (G1 y G3). Finalmente, los mencionados procedimientos de cómputo de distancias, el cual es un aspecto geométrico fuertemente ligado con estudios del grupo G2, vienen a reforzar y asimilar conceptos espaciales (norma L2, estadística no-paramétrica, etc.).

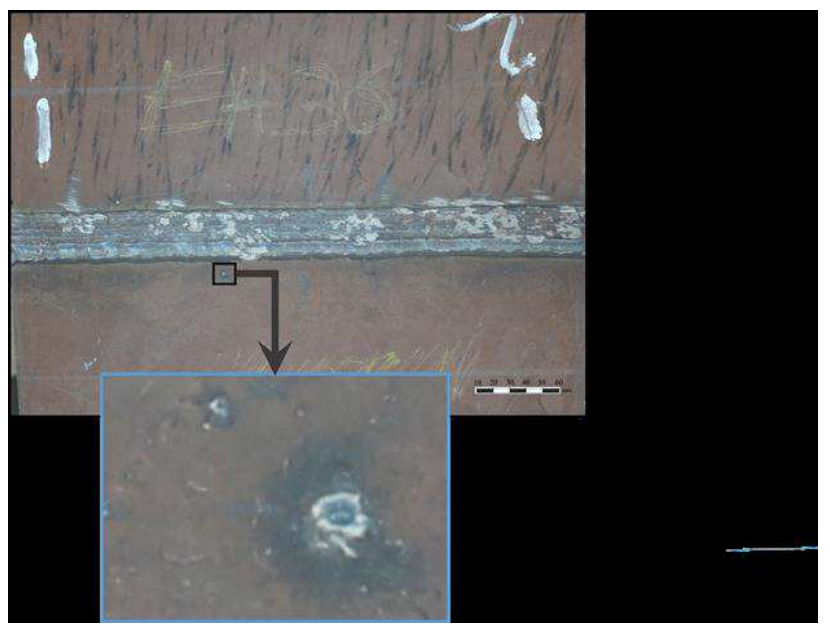

Figure 4: Fotografía de alta resolución de un especimen de soldadura disponible en laboratorio físico. Abajo: Detalle de una zona donde se hace presente un defecto de salpicadura menor de $1 \mathrm{~mm}$.

Concretamente, para el G2 se pueden suministrar actividades de mallado que permitan generar mallas sobre el modelo de nube de puntos, tal como se muestra en la (Fig. 7). Estas actividades pueden incluirse en el material bibliográfico para el G3 y también todos aquellos materiales 


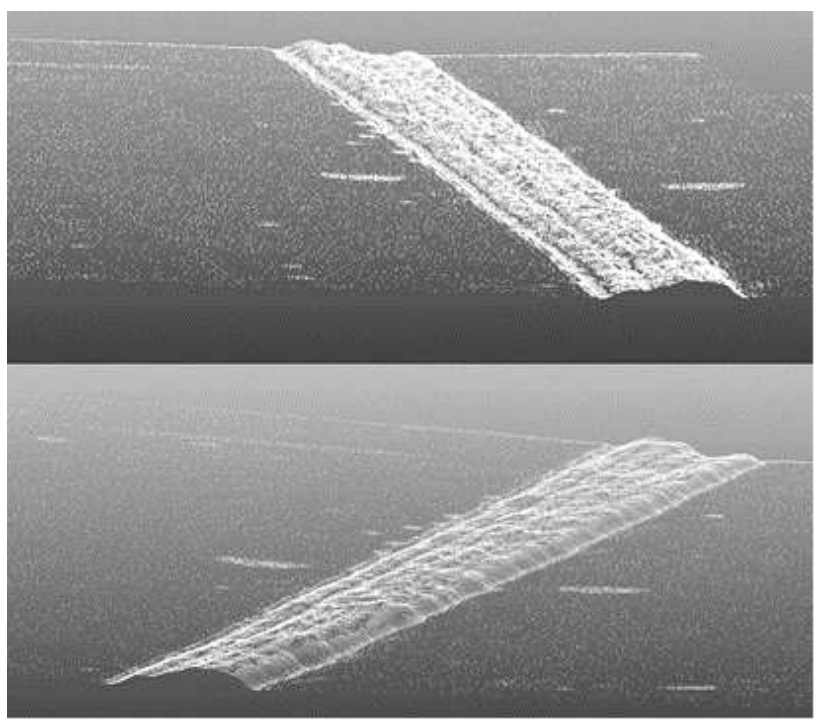

Figure 5: Modelo tridimensional fruto de la reconstrucción del especimen establecido en la Fig 4 utilizando ACMM.

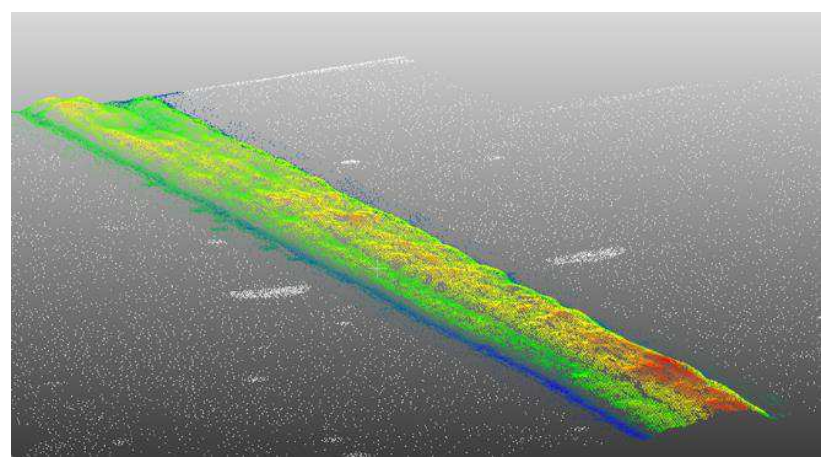

Figure 6: Generación de mapa de profundidad sobre el modelo de la Fig 5 siguiendo la metodología establecida en [Rodríguez-Martín et al, 2017].

teóricos que guíen al alumno durante el proceso.

\section{Conclusiones}

La contribución de este artículo se condensa en el planteam de una nueva metodología docente basada en las últimas investigaciones en el ámbito de la reconstrucción tridimensionales de modelos en soldadura publicadas en revistas de alto factor de impacto (Rodríguez-Martín et al, 2015; Rodríguez-Gonzálvez et al 2017; Rodríguez Martin et al, 2017;) que consiste en el diseño de paquetes de trabajo con modelos 3D de soldadura que permita adquirir a los alumnos de diferentes especialidades las competencias propias de las actividades de laboratorio en soldadura y geomática.

El paquete de trabajo consistirá en una colección de modelos tridimensionales que aportan la geometría del cordón de soldadura con alta precisión. Modelos que han sido generados con una herramienta avanzada de toma de datos

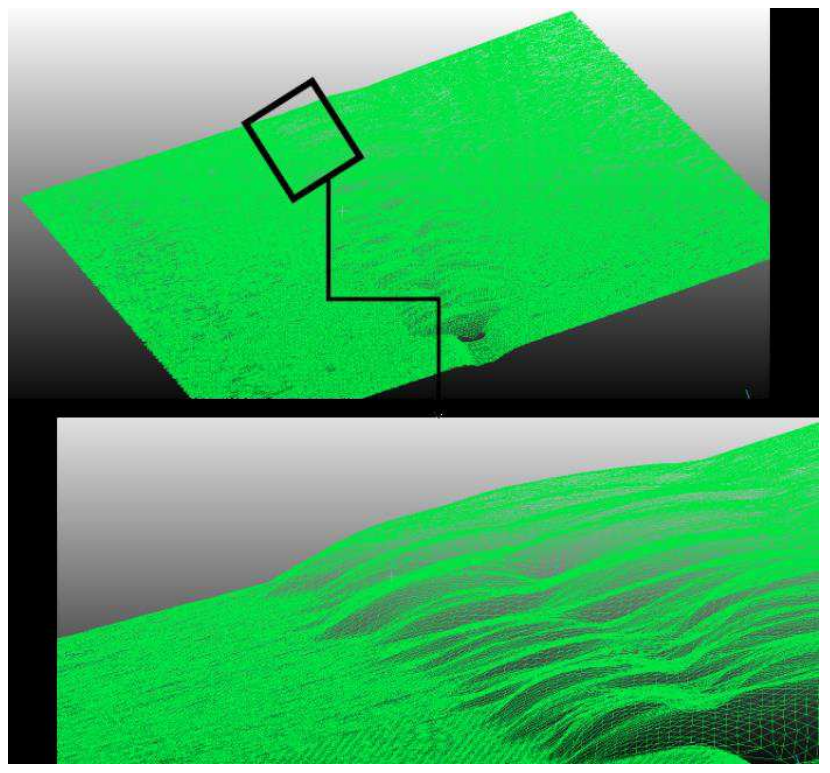

Figure 7: Generación de mapa de profundidad sobre el modelo de la Fig 5 siguiendo la metodología establecida en [Rodríguez-Martín et al, 2017].

geométricos y escaneo tridimensional como es un ACMM, garantizando la calidad reconstructiva y la precisión de los modelos. Un modelo tridimensional con geometría real permite el análisis de defectología sobre él mismo, siendo un material de experimentación flexible y de alto interés para alumnos de ingenierías del ámbito industrial, naval y aeroespacial, así como para la formación técnica de inspectores de soldadura en el ensayo tasado de Inspección Visual. Adicionalmente, se aporta en el paquete una colección de imágenes donde el alumno puede analizar las variedades cromáticas de los cordones de soldaduras y valorar aquellos defectos que no sean detectables por alteraciones geométricas sino por alteraciones cromáticas (e.g: las oxidaciones, las inclusiones de escoria, etc.).

Sin embargo, el objeto de este tipo de paquete de trabajo también puede ser de utilidad para alumnos de titulaciones relacionadas con la geomática donde, a partir de un mismo conjunto de datos iniciales (datos crudos capturado por el ACMM), se pueden generar diferentes productos finales según criterios tales como la fidelidad geométrica, la homogeneidad espacial o rapidez de visualización, entre otras. Dichas consideraciones entran de lleno en las competencias y destrezas a adquirir por parte de los titulados en los estudios relacionados con la geomática.

La manera de adaptar los paquetes de trabajo a cada especialidad, radicaría únicamente el material bibliográfico complementario. Mientras que a los alumnos del Grupo 1 y del Grupo 3 se les aportaría material para formación en inspección de soldaduras, así como la posible incorporación de los estándares internacionales que permiten la evaluación de las soldaduras; a los alumnos del Grupo 2 se les aportaría un material de estudio referente a las técnicas 
de mallado 3D, análisis de la propagación de errores desde la captura de los datos brutos hasta la generación de modelo 3D del espécimen de soldadura.

Nuevos estudios abordarán la medida del rendimiento en términos de aprendizaje de la innovación planteada. A este respecto se considera que la metodología experimental a abordar consistiría en una experimentación con grupo experimental (utilizando los paquetes de trabajo descrito) y un grupo de control (utilizando los especímenes de laboratorio tradicionales) y para medir el aprendizaje se utilizará pretest, postest y retención. A su vez nuevos estudios abordarán otros métodos de generación de modelos que permitan una texturizarían integrada, tales como fotogrametría o luz estructurada con un nivel de detalle suficientemente aceptable para la sustitución de los métodos tradicionales de enseñanza en inspección de soldadura.

\section{Referencias}

[1] American Welding Society. Examination book of specifications. (2006) (1st ed.). Miami, Fla.

[2] American Welding Society. The everyday pocket handbook for visual inspection and weld discontinuities - causes and remedies. (2004) (1st ed.). Miami, Fla.

[3] Calvo Verdú, M. (2006). Formación abierta y a distancia (1st ed.). Alcalá de Guadaira, Sevilla: Editorial Mad. Conde, A. (2013).

[4] Cerezo, F., Sastrón, F. (2015). Laboratorios Virtuales y Docencia de la Automática en la Formación Tecnológica de Base de Alumnos Preuniversitarios. Revista Iberoamericana De Automática e Informática Industrial RIAI 12(4): 419431. doi: 10.1016/j.riai.2015.04.005.

[5] CloudCompare V.2.6.6 (http://www.danielgm.net/cc/).

[6] Heradio, R., de la Torre, L., Galán, D., Cabrerizo, F.J., Herrera-Viedma, E., Dormido, S. (2016). Virtual and remote labs in education: a bibliometric analysis. Computers EG Education 98: 14-38. doi:10.1016/j.compedu.2016.03.010

[7] Mastmeyer, A., Wilms, M., Fortmeier, D., Schröder, J., Handels, H. (2016). Real-Time ultrasound simulation for training of us-guided needle insertion in breathing virtual patients. Studies in Health Technology and Informatics 220: 219-226. doi:10.3233/978-1-61499-625-5-219.

[8] Muñoz Nieto, A.L. (2014). Coordinación e intercambio de experiencias docentes en el marco del máster en geotecnologías cartográficas en ingeniería y arquitectura. Proyecto de innovación docente, Universidad de Salamanca, ID2014/0178.

[9] Osborne, B., Parange, N., Thoirs, K. (2015). The effectiveness of the use of high fidelity simulators in obstetric ultrasound training: A systematic review. Australasian Journal of Ultrasound in Medicine 18(3): 107-111.

[10] Rodríguez-Gonzálvez, P., Muñoz-Nieto, A.L., Izquierdo Álvarez, V., Almaraz Menéndez, F., Arias Pérez, B. (2018) Virtualización del máster en geotecnologías cartográficas en ingeniería y arquitectura. En Nuevo desafíos en la enseñanza superior (pp. 173-177). Ourense, España.

[11] Rodríguez-Gonzálvez, P., Rodríguez-Martín, M., Ramos, L., González-Aguilera, D. (2017). 3D reconstruction methods and quality assessment for visual inspection of welds. Automation In Construction 79: 49-58. doi:10.1016/j.autcon.2017.03.002.

[12] Rodriguez-Martín, M., Lagüela S., Gonzalez-Aguilera, D., Rodríguez-Gonzalvez, P. (2015). Procedure for quality inspection of welds based on macro-photogrammetric threedimensional reconstruction. Optics \& Laser Technology $\mathbf{7 3}$ 54-62. doi:10.1016/j.optlastec.2015.04.011.

[13] Rodríguez-Martín, M., Rodríguez-Gonzálvez, P., González-
Aguilera, D. Fernández-Hernández, J. (2017). Feasibility study of a structured light system applied to welding inspection based on articulated coordinate measure machine data. IEEE Sensors Journal. doi:10.1109/JSEN.2017.2700954.

[14] Rossiter, J. (2017). Low production cost virtual modelling and control laboratories for chemical engineering students. In International Federation of Automatic Control 20th IFAC Symposium on Automatic Control in Aerospace-ACA 2016. Quebec.

[15] Vergara, D., Nuñez, F., Ferrer, J., Rodríguez-Martín, M. (2016). Teaching of ultrasound test by means of virtual reality. In TeLe(In)2 Teaching \& Learning Innovation Institute Conference (pp. 9-12). Madrid. Retrieved from https://telein2.wordpress.com/publications/

[16] Vergara, D., Rubio, M.P., Prieto, F. (2014). Nueva herramienta virtual para la enseñanza de la caracterización mecánica de materiales. Revista de Educación en Ingeniería 9(17): 98-107.

[17] Welding and allied processes - Classification of geometric imperfections in metallic materials - Part 1: Fusion welding. European Committee for Standardization. EN-ISO 65201:2007 (2007).

[18] Welding. Fusion-welded joints in Steel, nickel, titanium and their alloys (beam welding excluded). Quality levels for imperfections (ISO 5817:2003 corrected version.2005, including Technical Corrigendum). European Committee for Standardization. EN-ISO-5817: 2009 (2009). 